\title{
Using a Cluster-Based Regime-Switching Dynamic Model to Understand Embodied Mathematical Learning
}

\author{
$\mathrm{Lu} \mathrm{Ou}^{*}$ \\ ACTNext \\ ACT, Inc. \\ Iowa City, Iowa \\ lu.ou@act.org
}

\author{
Alejandro Andrade \\ ACTNext \\ ACT, Inc. \\ Iowa City, Iowa \\ andradea@act.org
}

\author{
Rosa Alberto \\ Mathematics \\ Utrecht University \\ Utrecht, the Netherlands \\ r.a.alberto@uu.nl
}

\author{
Gitte van Helden \\ Mathematics \\ Utrecht University \\ Utrecht, the Netherlands \\ gittevanhelden@gmail.com
}

\author{
Arthur Bakker \\ Mathematics \\ Utrecht University \\ Utrecht, the Netherlands \\ a.bakker4@uu.nl
}

\begin{abstract}
Embodied learning and the design of embodied learning platforms have gained popularity in recent years due to the increasing availability of sensing technologies. In our study, we made use of the Mathematical Imagery Trainer for Proportion (MIT-P) that uses a touchscreen tablet to help students explore the concept of mathematical proportion. The use of sensing technologies provides an unprecedented amount of high-frequency data on students' behaviors. We investigated a statistical model called mixture RegimeSwitching Hidden Logistic Transition Process (mixRHLP) and fit it to the students' hand motion data. Simultaneously, the model finds characteristic regimes and assigns students to clusters of regime transitions. To understand the nature of these regimes and clusters, we explore some properties in students' and tutor's verbalization associated with these different phases.
\end{abstract}

\section{CCS CONCEPTS}

- Computing methodologies $\rightarrow$ Modeling methodologies; • Applied computing $\rightarrow$ Interactive learning environments; Computer-assisted instruction; • Human-centered computing $\rightarrow$ Empirical studies in collaborative and social computing.

\section{KEYWORDS}

Multimodal Learning Analytics, Embodied Cognition, Mathematical Learning, Dynamic Models

\section{ACM Reference Format:}

$\mathrm{Lu} \mathrm{Ou}$, Alejandro Andrade, Rosa Alberto, Gitte van Helden, and Arthur Bakker. 2020. Using a Cluster-Based Regime-Switching Dynamic Model to Understand Embodied Mathematical Learning. In Proceedings of the 10th International Conference on Learning Analytics and Knowledge (LAK'20),

${ }^{*}$ Both authors contributed equally to this research.

Permission to make digital or hard copies of part or all of this work for personal or classroom use is granted without fee provided that copies are not made or distributed for profit or commercial advantage and that copies bear this notice and the full citation on the first page. Copyrights for third-party components of this work must be honored.

For all other uses, contact the owner/author(s).

LAK '20, March 23-27, 2020, Frankfurt, Germany

(c) 2020 Copyright held by the owner/author(s).

ACM ISBN 978-1-4503-7712-6/20/03.

https://doi.org/10.1145/3375462.3375513
March 23-27, 2020, Frankfurt, Germany. ACM, New York, NY, USA, 6 pages. https://doi.org/10.1145/3375462.3375513

\section{INTRODUCTION}

Embodied learning and the design of embodied learning platforms have gained popularity lately, in part, due to the increasing availability of sensing technologies, such as touchscreen tablets, which allow for a more natural interaction than traditional mouse-and-keyboard interfaces. Embodied learning builds upon theories of embodied cognition, which argue that abstract concepts are fundamentally grounded in, or emerge from, physical and time-constrained experiences with our environment [16, 21]. Learning is, therefore, a process of dynamically coordinating modalities that lead to direct or metaphorical abstract conceptualizations of the world [2-4, 12].

Our study develops upon the works of Dor Abrahamson and colleagues who have studied the development of the mathematical concept of proportionality. To ground the learning of proportionality on its physical enactment, Abrahamson and colleagues developed an embodied learning platform called the Mathematics Imagery Training of Proportion (MIT-P) [3, 10]. A recent version of the MIT-P developed by the embodied cognition group at the University of Utrecht uses a touchscreen tablet to help students explore the concept of proportion physically (see Figure 1). The tablet displays two vertical bars whose heights are controlled by the participant's index fingers. The bars change color from red to green any time they achieve a designated ratio (e.g., 1:2), which is initially unknown to the student. During an instructional activity, the student needs to find this hidden rule that makes the bars green. They move the bars up and down until they find places where the bars turn green. After they find the first green, they need to keep the bars green all the time while moving them. As students freely explore different configurations, a tutor coaches them to reflect upon the activity. Besides, the instructor progressively introduces two frames of reference to the interaction field, such as an equallyspaced grid and numerals. As students adopt these frames to keep the bars green and explain their movements, they shift into more expert ways of seeing, moving, and talking. 

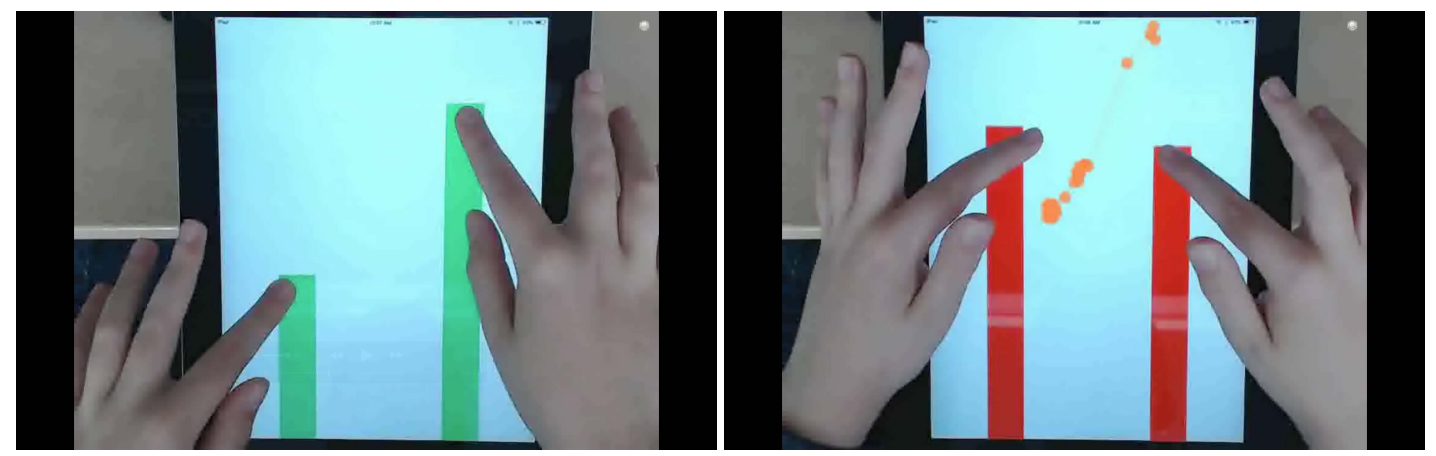

Figure 1: Touchscreen version of the MIT-P. The bars turn green when the ratio between the bar heights is 1:2, otherwise the bars color turns red.

Using the MIT-P, researchers have advanced arguments as to how action-based learning environments foment spontaneous coordination of sensorimotor activity, which, in turn, supports the learning of mathematical concepts [17]. For instance, various studies with the MIT-P have investigated relevant changes in students' sensorimotor coordination and have advanced hypotheses as to how the development of dynamic coordination represents the learning of the concept of proportion $[1,10]$. In particular, using qualitative approaches, researchers have found empirical evidence of various patterns of coordination dynamics. They have discovered six solution strategies: fixed interval, changing interval, additive (two kinds), multiplicative, and speeds. These embodied strategies outline a learning trajectory of the concept of proportion. In the beginning, students start by exploring the height of the bars in random ways. They then maintain a fixed interval between the bars (similar to the conceptual errors as mentioned above), or switch towards a varying distance, before realizing that one bar's height is half/double of the other. With the inclusion of the grid, students shift towards a step-wise method where they move one unit with the left hand and two with the right hand. Finally, with the introduction of the numerals, students start making a greater emphasis on the pairs 1-2, 2-4, 3-6, and so on. Moreover, researchers argue that students' deployment of additive strategies contributes as one primary source to a misconception of proportionality. For example, students often believe that $2: 3$ is equal to $3: 4$ and $4: 5$ because the two numbers in each ratio have a difference of one [13]. Fuson and Abrahamson [11] found that this misconception correlates with students' difficulties in physically enacting the proportional progression with their hands.

In the qualitative approaches, researchers have faced various drawbacks, such as dealing with hours of video to review, the need to train and retrain our qualitative judgments to produce a reliable coding scheme, and the difficulty in spotting variability between students to form groups (i.e., clusters of students with similar sequences of behaviors). The goal of the current study is to quantitatively analyze the log files of hand data to uncover the presence of phases in students' sequences of behaviors and to understand whether there are patterns of phase transitions across students that are otherwise difficult to detect using a qualitative analysis.

If we could identify model-based clusters and phase transitions based on observation data, then in real-time monitoring of students' learning progress, we could assign students into different clusters and make model-based predictions of their behavior. These analytical results would benefit educators and educational designers who want to tailor instructions to the specific needs of groups of students or want to shift students from one cluster to another. Also, a statistical model of both within- and between-subject qualitative and quantitative variability would support rich theoretical insights. For example, we might want to understand whether students who learn to coordinate their hands quickly also conceptualize better; or whether all students need first to struggle and overcome these physical coordination difficulties to have better conceptualizations.

In summary, our research questions are 1) are there distinct handmovement regimes and regime transitions in students' learning processes? 2) what properties do the regimes have? 3) are there clusters of students who share similar patterns of regime-switching learning processes? 4) What do these regimes and clusters reveal about proportionality learning?

Sensing technologies such as the MIT-P provide an unprecedented amount of data about the momentary changes in students' behaviors. Although some time-series methods allow for the modeling of quantitative within-subject changes (e.g., in behaviors), most of these methods do not simultaneously account for possible group differences across students and phase transitions over time. We need more sophisticated methods to analyze both the quantitative and qualitative variability (i.e., group and phase differences) in students' interaction with their learning environments.

In this paper, we investigate a statistical model called mixture Regime-Switching Hidden Logistic Transition Process (mixRHLP) and fit it to the hand movement data. The model fitting allows us to retrieve characteristic movement regimes (or phases) from hand movement changes. Simultaneously, the model could help identify characteristic regime transitions and assign students to a particular cluster. Then, to assess the relation between hand coordination and conceptualization, we explore students' and tutor's verbalization associated with these regimes. According to the embodied learning theory, how students coordinate their hand movements is related to their level of understanding of the to-be-learned mathematical concept. That is, the better the hand movement embody the mathematical concept, the more verbal references to proportionality there would be. In a similar vein, we hypothesize that the tutor adapts her 
verbal guidance in response to how a student moves their hands to support their learning better.

Our analysis showed that there were different patterns of hand movements in various phases of students' learning proportions using the MIT-P. Moreover, we identified two distinct clusters of students with characteristic phase transitions of hand movement patterns that can be associated with slow- and quick-learners. Furthermore, we found that there are different characteristics of studenttutor verbal communication during different learning phases.

Our results contribute to the field of embodied learning by analyzing quantitative data on sensorimotor activity and student-tutor conversations. The work should be of broad interest to the learning analytics community. First, the MIT-based embodied design has already extended to domains beyond proportionality. They include but not limited to linear functions, parabola, area, trigonometric functions, and angles. Our current work inspires empirical research in each domain to contribute to our understanding of embodied learning. Second, the statistical method we present applies to any time-series data, including the increasingly accessible multimodal data that shed light on our understanding of learning and learning environments. Last but not least, the statistical model simultaneously represents both between- and within-subject qualitative and quantitative variability that is prevalent in students' learning processes. It extends many traditional models, including finite mixture models [8, 9], hidden Markov models [5, 20], and regime-switching dynamic models [7,14], to enable crucial inferences of students' learning processes to inform interventions.

\section{METHODS}

\subsection{Data Sources}

We conducted a secondary data analysis from a previous study [10]. In the study, forty-five fifth- and sixth- graders of ages 9-11 $(\mathrm{M}=135.37$ months, $\mathrm{SD}=8.37$ months, and Female $=17)$ participated in task-based semi-structured interviews at schools in the Netherlands. Screen recordings of the activity, participants' hand movement data in the form of bar height coordinates, eye-tracking data, and audio data were captured during each interview. Due to technical problems, data from only 37 participants were included in the current analysis.

During the interview with the MIT-P, the students' goal is to find a hidden rule that makes the bars green. The students may move the bars up and down and see how the colors of the bars change. After they find the first green, they are asked to keep the bars green while moving them. As the student freely explores the interface, the tutor coaches the student to reflect upon the activity and think aloud. Besides, the tutor progressively introduces two frames of reference to the interaction field, such as an equally-spaced grid with and without numerals overlaid on the screen, and different tasks with varying proportion values in the hidden rules.

However, we did not aim to analyze all the data collected during the various tasks and with different pedagogical aids. Instead, we limited our analysis to the task corresponding to the 1:2 proportion and only focused on the part of the task where the tutor uses a blank background, without grids or numerals overlaid on the screen. We may analyze additional data under different task and background conditions in future studies. The average time spent in these blankbackground segments was 7.3 minutes.

\subsection{Model Assumptions}

We mathematically represented the time-series data of right and left hand ratios using the mixture Regime-Switching Hidden Logistic Transition Process (mixRHLP) model $[6,15]$. Suppose for each student $i, i=1,2,3, \cdots, N$, there are a total of $T$ measurement occasions and $T$ measurements, respectively denoted as $T \times 1$ vectors of $\boldsymbol{t}=\left(t_{j}\right)$ and $\boldsymbol{y}_{i}=\left(y_{i}\left(t_{j}\right)\right), j=1,2,3, \cdots, T$. We assume $\boldsymbol{y}_{i}$ follow a mixture distribution, whose density $p(\cdot)$ is a weighted sum of component densities $p_{k}(\cdot)$ as

$$
p\left(\boldsymbol{y}_{i} \mid \boldsymbol{t}_{i} ; \boldsymbol{\Theta}\right)=\sum_{k}^{K} P\left(Z_{i}=k\right) p_{k}\left(\boldsymbol{y}_{i} \mid \boldsymbol{t}_{i}, Z_{i}=k\right),
$$

where $Z_{i} \in\{1,2, \cdots, K\}$ denotes student $i$ 's latent cluster class, with $\alpha_{i k} \triangleq P\left(Z_{i}=k\right)$ being the probability of student $i$ belonging to latent cluster class $k . \Theta_{\boldsymbol{k}}$ contains all parameters in the component density $p_{k}(\cdot)$, and $\Theta$ contain all parameters in the density $p(\cdot)$.

At each time point $t_{j}$, we further assume $y_{i}\left(t_{j}\right)$ follows a finite Gaussian mixture regression model, whose component density is normally distributed with mean $\boldsymbol{X}_{j} \boldsymbol{\beta}_{k r}$ and a variance of $\sigma_{k r}^{2}$, denoted as $\left.\mathcal{N}\left(\boldsymbol{X}_{j} \boldsymbol{\beta}_{k r}, \sigma_{k r}^{2}\right)\right)$, where $r=1,2, \cdots, R_{k}, \boldsymbol{\beta}_{k r}$ is a $(d+$ 1) $\times 1$ vector of regression coefficients, and the design matrix $X_{j}$ is $\left[\begin{array}{llll}t_{j}^{0} & t_{j}^{1} & \cdots & t_{j}^{d}\end{array}\right]$, if the order of the polynomial regression is $d$.

If we assume $\boldsymbol{y}_{i} \mid \boldsymbol{t}_{i}, Z_{i}=k$ are serially independent, $p_{k}(\cdot)$ can be written as

$$
p_{k}\left(\boldsymbol{y}_{i} \mid \boldsymbol{t}_{i}, Z_{i}=k\right)=\prod_{j=1}^{T} \sum_{r}^{R_{k}} P\left(H_{i j}=r \mid t_{j}, Z_{i}=k\right) \mathcal{N}\left(\boldsymbol{X}_{j} \boldsymbol{\beta}_{k r}, \sigma_{k r}^{2}\right),
$$

where $H_{i j} \in\left\{1,2, \cdots, R_{k}\right\}$ denotes student $i$ 's latent regime at time $t_{j}$ and is assumed to follow a multinomial logistic regression model such that the probability of student $i$ belonging to latent regime $r$ at time $t_{j}$ under the condition that student $i$ belongs to latent cluster class $k$ is

$$
P\left(H_{i j}=r \mid t_{j}, Z_{i}=k\right)=\frac{\exp \left(\omega_{k r 0}+\omega_{k r 1} t_{j}\right)}{\sum_{s=1}^{R_{k}} \exp \left(\omega_{k s 0}+\omega_{k s 1} t_{j}\right)}
$$

with $\omega_{k s 0}=\omega_{k s 1}=0$ in the reference class. The regression coefficients $\omega_{k}=\left[\begin{array}{ll}\omega_{k r 0} & \omega_{k r 1}\end{array}\right]$ control the regime transitions.

Assuming the observed data across subjects $\boldsymbol{y}_{i}, i=1,2,3, \cdots, N$ are independently identically distributed, we can write the loglikelihood function of $\boldsymbol{\Theta}$ given all observed data $Y \stackrel{\Delta}{=}\left[\boldsymbol{y}_{i}\right]$ as

$$
l(\boldsymbol{\Theta})=\log \prod_{i}^{N} p\left(\boldsymbol{y}_{i} \mid \boldsymbol{t}_{i} ; \boldsymbol{\Theta}\right)=\sum_{i}^{N} \log \sum_{k}^{K} \alpha_{i k} p_{k}\left(\boldsymbol{y}_{i} \mid \boldsymbol{t}_{i}, Z_{i}=k\right) .
$$

Thus, the parameters in the model can be estimated using maximum likelihood approaches.

\subsection{Talk Analysis}

We transcribed the audio data and aligned the spoken utterances to the video using the ELAN software [18]. As the conversation was originally in Dutch, an English translation was produced using the 
Google Translation API and then checked for clarity by one of the researchers. Then, we labeled each utterance with its corresponding regime. We analyzed the talk patterns by looking at the distribution of words and the most frequent n-grams in each regime. An n-gram analysis consists of running a sliding window of size $n$ through the text and extracting the sequences of words within that window [19]. Specifically, we looked at 3-, 4-, and 5-grams-i.e., sequences of three, four, and five words. Then, we sorted the sequences in descending order of counts to find out the most frequent n-grams in each regime.

\section{RESULTS}

\subsection{Cluster-Based Regime-Switching Dynamics of Hand Movements}

Using the R package mixRHLP [6, 15], we fitted the mixRHLP model to the time-series data of the right and left hand ratios with different values of the number of clusters (1-4), the number of regimes (1-4), and the order of polynomial regressions (1-2). Among all 32 models, we chose the simpler model in the list of models with the smallest three BIC values, which consisted of two clusters, three regimes, and linear regressions.

The parameter estimates from fitting the chosen model to the data are summarized in Table 1 . In Regime 1, the regression intercepts were near one with small error variances, indicating that hands were moving at the same height. In Regime 2, the intercepts were still around one, but the error variances were larger, indicating hands moving with noticeable variability. In Regime 3, the intercepts were about two with small error variances, suggesting hands moving at the desired heights of 1:2 ratio to keep the bars green. Indeed, Regime 3 was the desired outcome of this activity since the hands would maintain a 1:2 ratio - for example, one at a value of 2 and the other at a value of 4 . Additionally, Figure 2 illustrates the estimated expected logistic curves of the probabilities of an individual being in a regime during the interview. The probability of an individual being in Cluster 1 was estimated to be .42 , a little smaller than that of being in Cluster 2. In Cluster 1, the probability of being in Regime 1 was the highest at the start of the session, but quickly transitioned into the probability of being in Regime 2; the probability of being in Regime 3 became the highest at around $70 \%$ into the interview session. In Cluster 2, the probability of being in Regime 1 was the highest until approximately $10 \%$ into the session, when the probability of being in Regime 2 took the lead; then, Regime 3 became the most probable state at about $20 \%$ into the session, much sooner compared to Cluster 1 . Indeed, what the logistic curves tell us is that a student in Cluster 1 has about the same likelihood to find the rule than not to find it, as indicated by the high logistic curve of Regime 2 for most of the task segment. Instead, students in Cluster 2 have a much higher probability of finding the proportional rule, especially after the first half. It is apparent that, in Cluster 2, the probability of being in Regime 1 goes down quickly and almost disappears after the first quarter. On the other hand, the probability of Regime 2 goes down but still lingers on, albeit low, until the end of the task segment. For example, Figure 3 shows two characteristic phase transitions that are representative of these two clusters; one for Participant 75 and the other for Participant 83, who displayed different hand movement dynamics but were randomly chosen for illustration. Participant 75 , classified in Cluster 1, spent a substantial proportion (>50\%) of the session exploring various ratios or only moving her hands at the same speed. Participant 83, classified in Cluster 2, spent only $10 \%$ of the time moving hands at the same heights and quickly switched to a 1:2 ratio phase (Regime 3), interspersed with chunks of short exploration phases (Regime 2).

\subsection{Students' Verbalization}

Results show that the content of the talk was different between regimes, as illustrated by the most frequent n-grams (see Table 2). During Regime 1, most of the talk was descriptive, such as "I get a little bit of green at the bottom of the screen." During Regime 2 , when there was much exploration of different hand ratios, the talk contains some explanation attempts which are often not very specific or depict confusion. For instance, this search was accompanied by utterances such as "This one is higher than the other" or with confusion utterances such as "I do not know." During Regime 3 , where the hands achieved a 1:2 ratio, most of the utterances explained the possible reasons. For instance, students frequently used proportional comparisons with utterances such as "Both are light green when the right bar is twice as high as the left," or "They are green if the left is half of the right."

\subsection{Tutor's Verbalization}

Results showed that the tutor's verbalization had particular characteristics depending on the regime (see Table 2). Specifically, the talk during Regime 1 consisted of the tutor introducing the task to the student (e.g., "I want you to find green in as many ways as you can, and I want you to say everything you think out loud."). During Regime 2, the tutor's talk prompted active inquiry and reflection, such as "What do you mean by?", "Can you show that?", "What do you notice?". During Regime 3, the talk inquired about a student's ideas as well as encouraged them to further exploration and find a global rule or movement (e.g., "How did you do that?", "Do you think the bars will turn green here?").

\section{DISCUSSION AND CONCLUSION}

In this study, we examined a group of fifth and sixth-grade students' engagement in an action-based learning environment where they explored the concept of proportion by moving their hands on a touchscreen. We collected data in the form of hand logs and conducted a statistical analysis of the hands' ratio and discovered qualitative differences during the activity and across students. We characterized the between-subjects variation as clusters and the within-subject changes as regimes. In different regimes, we identified interesting patterns of hand movements. In particular, regimes 2 and 3 were indicative of important cognitive activities, such as exploration and consolidation. Clustering allowed us to characterize students by how they approach the task. For instance, whereas some students spent large segments of the task exploring various hands' ratios, other students spent most of their time reflecting and advancing explanations as to why the bars turned green.

More specifically, we interpret our results for the three regimes as follows. Regime 1 corresponds to an initial phase of the embodied interaction. During this regime, the hands were at the same height; 
Table 1: Parameter estimates from the empirical example

\begin{tabular}{|c|c|c|c|c|c|c|c|c|}
\hline & & \multirow[b]{2}{*}{$X$} & \multicolumn{2}{|c|}{$\begin{array}{l}\text { Regime } 1 \\
\text { Moving at the same height }\end{array}$} & \multicolumn{2}{|c|}{$\begin{array}{c}\text { Regime } 2 \\
\text { Moving with large variability }\end{array}$} & \multicolumn{2}{|c|}{$\begin{array}{l}\text { Regime } 3 \\
\text { Moving at the desired ratio }\end{array}$} \\
\hline & & & 1 & $t$ & 1 & $t$ & 1 & $t$ \\
\hline \multirow{3}{*}{$\begin{array}{c}\text { Cluster } 1 \\
\text { Slow-discovering } \\
\text { students }\end{array}$} & \multirow{3}{*}{$\alpha_{1}=.42$} & $\omega_{1}$ & 1.899 & -7.871 & 1.864 & -2.597 & 0 & 0 \\
\hline & & $\boldsymbol{\beta}_{1}$ & 1.014 & -0.019 & 1.149 & 0.016 & 2.017 & -0.061 \\
\hline & & $\sigma_{1}^{2}$ & 0.003 & & 0.180 & & 0.015 & \\
\hline \multirow{3}{*}{$\begin{array}{c}\text { Cluster } 2 \\
\text { Quick-discovering } \\
\text { students }\end{array}$} & \multirow{3}{*}{$1-\alpha_{1}=.58$} & $\omega_{2}$ & 1.423 & -14.651 & 0 & 0 & -0.386 & 1.794 \\
\hline & & $\boldsymbol{\beta}_{2}$ & 1.018 & -0.039 & 0.896 & 0.594 & 2.027 & -0.043 \\
\hline & & $\sigma_{2}^{2}$ & 0.009 & & 0.213 & & 0.009 & \\
\hline
\end{tabular}

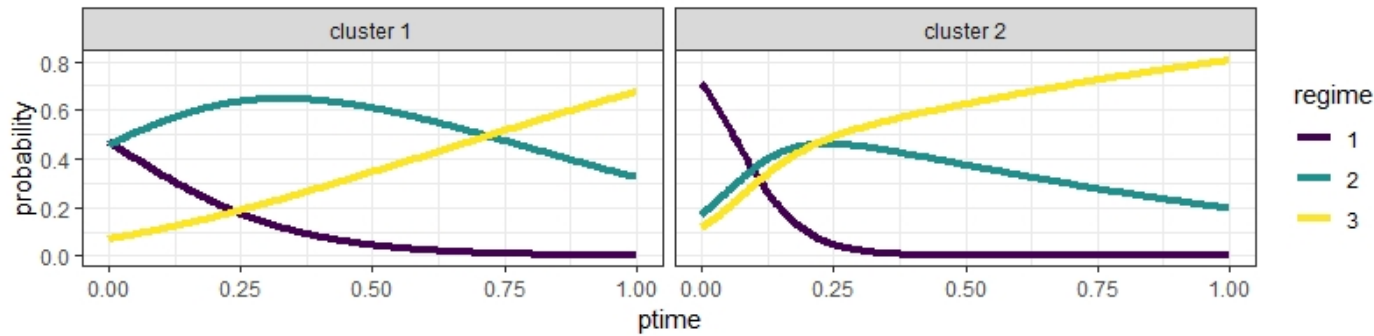

Figure 2: Estimated logistic curves of the three regimes within the two clusters.

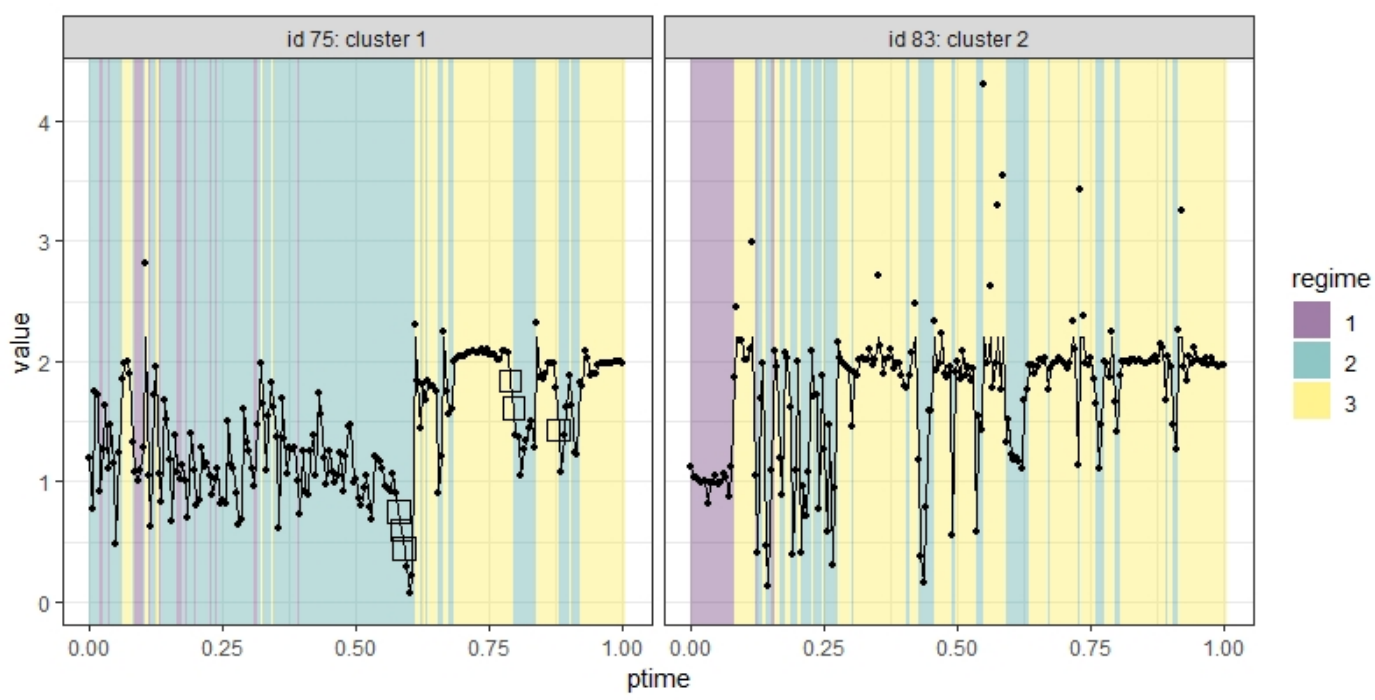

Figure 3: Characteristic time-series data for each of the two clusters. Left: Participant 74 represents a characteristic Cluster 1, with a lot of exploration (Regime 2) and a final convergence to the right ratio (Regime 3 ) around the final third of the task. Right: Participant 83 represents a characteristic Cluster 2, with a faster convergence to the 1:2 ratio (Regime 3) but with many interspersed exploration moments (Regime 2).

perhaps the student awaited to see what happens next. Regime 2 corresponds to an intermediate phase of the interaction. During this regime, the student was actively exploring different hand ratios, perhaps attempting to find how to make the bars green. From prior qualitative observations, we knew that during this regime, students might deploy a mixture of strategies where two hands move independently or move at fixed distances. However, our mixRHLP model of hands' ratio misses this distinction, resulting in one of the limitations of our current approach. Regime 3 corresponds to a later phase of the interaction. During this phase, the hands maintained at a 1:2 ratio. However, as the tutor asked students to find green in as 
Table 2: Students(S)' and Tutors(T)’ Most Frequent N-Grams

\begin{tabular}{|c|c|c|c|}
\hline N-Grams & Regime 1 & Regime 2 & Regime 3 \\
\hline 3 & $\begin{array}{l}\text { S: "I have them" } \\
\text { T: "can you find", "can you say" }\end{array}$ & $\begin{array}{l}\text { S: "at same time" } \\
\text { T: "do you notice" }\end{array}$ & $\begin{array}{l}\text { S: "they turn green" } \\
\text { T: "bars turn green" }\end{array}$ \\
\hline 4 & $\begin{array}{l}\text { S: "at bottom of screen" } \\
\text { T: "you think out loud", "what } \\
\text { you are doing" }\end{array}$ & $\begin{array}{l}\text { S: "here again here again", "I do } \\
\text { not know" } \\
\text { T: "what do you mean", "can } \\
\text { you show that" }\end{array}$ & $\begin{array}{l}\text { S: "right bar is now", "it both are } \\
\text { light" } \\
\text { T: "find even more green", "at } \\
\text { bottom of screen" }\end{array}$ \\
\hline 5 & $\begin{array}{l}\text { S: "a little bit of green" } \\
\text { T: "as many ways as possible", "every- } \\
\text { thing you think out loud" }\end{array}$ & $\begin{array}{l}\text { S: "the right bar is higher", "if I do } \\
\text { that for" } \\
\text { T: "what do you have to do", "hands } \\
\text { at the same time" }\end{array}$ & $\begin{array}{l}\text { S: "bar is twice as high", "they both } \\
\text { are light green" } \\
\text { T: "how did you do that?", "find } \\
\text { green at the top" }\end{array}$ \\
\hline
\end{tabular}

many ways as possible, from time to time, this particular ratio was lost, and the student fell back into a new exploration phase (Regime 2). Note that to keep the same ratio as the hands move up, one hand has to move twice as fast as the other hand. It is challenging to students even though they have figured out the proportion rule.

Our cluster-based regime-switching analysis has some limitations. First, our current analysis only took into account the hands' ratio, but there are other hand-movement variables such as speed and distance between hands that we could have utilized. Second, we constrained the analysis to the hand logs, but we can further explore the addition of the eye gaze data and study how hand and eye movements coordinate in such activities. Third, we only analyzed the interview segment with a blank background for the 1:2 ratio. Students' strategy may change when the grid and numerals show up. Finally, we still need to conduct an in-depth analysis of the clusters and regimes to fully understand their properties and how they relate to the process of learning of proportion.

Despite its limitations, this study furthers our knowledge of how students' mathematical concepts may build on their physical experiences. Furthermore, we illustrated how to apply the mixRHLP model to extracting qualitative patterns from quantitative time-series of human behavior. Hence, we contribute to the field of studying human dynamics beyond mathematics learning and teaching.

Last but not least, we anticipate some applications of this work. For instance, in collaborative learning environments such as classrooms, we might be able to monitor students' real-time behavior and utilize the technique illustrated in this paper to generate learners' profiles and tailor personalized and group-based feedback to facilitate learning.

\section{REFERENCES}

[1] Dor Abrahamson, Rosa G. Lee, Andrea G. Negrete, and José F. Gutiérrez. 2014 Coordinating visualizations of polysemous action: Values added for grounding proportion. ZDM - International fournal on Mathematics Education 46, 1 (2014), 79-93. https://doi.org/10.1007/s11858-013-0521-7

[2] Dor Abrahamson and Raúl Sánchez-García. 2016. Learning is moving in new ways: The ecological dynamics of mathematics education. Journal of the Learning Sciences 25, 2 (2016), 203-239.

[3] Dor Abrahamson, Shakila Shayan, Arthur Bakker, and Marieke van der Schaaf 2015. Eye-tracking Piaget: Capturing the emergence of attentional anchors in the coordination of proportional motor action. Human Development 58, 4-5 (2015), 218-244. https://doi.org/10.1159/000443153

[4] Luis Alejandro Andrade-Lotero. 2018. Elicited Gestures: An Embodied Instructional Design to Support the Understanding of Quantitative Patterns of Complex Systems. Ph.D. Dissertation.
[5] Meirav Arieli-Attali, Lu Ou, and Vanessa R. Simmering. 2019. Understanding test takers' choices in a self-adapted test: A hidden Markov modeling of process data. Frontiers in Psychology 10, FEB (2019), 1-15. https://doi.org/10.3389/fpsyg.2019. 00083

[6] F. Chamroukhi, D. Trabelsi, S. Mohammed, L. Oukhellou, and Y. Amirat. 2013. Joint segmentation of multivariate time series with hidden process regression for human activity recognition. Neurocomputing 120 (November 2013), 633-644. https://chamroukhi.com/papers/chamroukhi_et_al_neucomp2013b.pdf

[7] Sy-Miin Chow, Lu Ou, Arridhana Ciptadi, Emily Prince, Dongjun You, Michael D. Hunter, James M. Rehg, Agata Rozga, and Daniel S. Messinger. 2018. Representing sudden shifts in intensive dyadic interaction data using differential equation models with regime switching. Psychometrika 83, 2 (2018), 476-510. https: //doi.org/10.1007/s11336-018-9605-1

[8] Craig R. Colder, Richard T. Campbell, Erin Ruel, Jean L. Richardson, and Brian R. Flay. 2002. A finite mixture model of growth trajectories of adolescent alcohol use: Predictors and consequences. Fournal of Consulting and Clinical Psychology 70, 4 (2002), 976-985. https://doi.org/10.1037/0022-006X.70.4.976

[9] Conor V. Dolan, Brenda R. Jansen, and Han L. J. Van der Maas. 2004. Constrained and unconstrained multivariate normal finite mixture modeling of Piagetian data. Multivariate Behavioral Research 39, 1 (2004), 69-98. https://doi.org/10.1207/ s15327906mbr3901 3

[10] Carolien A.C.G. Duijzer, Shakila Shayan, Arthur Bakker, Marieke F. Van der Schaaf, and Dor Abrahamson. 2017. Touchscreen tablets: Coordinating action and perception for mathematical cognition. Frontiers in Psychology 8, FEB (2017), 63-81. https://doi.org/10.3389/fpsyg.2017.00144

[11] Karen C Fuson and Dor Abrahamson. 2005. Understanding ratio and proportion as an example of the apprehending zone and Conceptual-Phase problem-solving models. Handbook of Mathematical Cognition (2005), 213-234.

[12] George Lakoff and Rafael E Núñez. 2000. Where mathematics comes from: How the embodied mind brings mathematics into being. AMC 10, 12 (2000), 720-733.

[13] Susan J Lamon. 2007. Rational numbers and proportional reasoning: Toward theoretical framework for research. Second Handbook of Research on Mathematics Teaching and Learning 1 (2007), 629-667.

[14] Lu Ou, Michael D Hunter, and Sy-Miin Chow. 2019. What's for dynr: A package for linear and nonlinear dynamic modeling in R. The R fournal 11, 1 (2019), 1-20.

[15] A. Samé, F. Chamroukhi, Gérard Govaert, and P. Aknin. 2011. Model-based clustering and segmentation of time series with changes in regime. Advances in Data Analysis and Classification 5 (2011), 301-321. Issue 4. https://chamroukhi. com/papers/adac-2011.pdf

[16] Lawrence Shapiro. 2019. Embodied Cognition. Routledge.

[17] Shakila Shayan, Dor Abrahamson, Arthur Bakker, ACG Duijzer, and MF Van der Schaaf. 2015. The emergence of proportional reasoning from embodied interaction with a tablet application: An eyetracking study. In Proceedings of the 9th international technology, education, and development conference (INTED 2015). 5732.

[18] Han Sloetjes and Peter Wittenburg. 2008. Annotation by category-ELAN and ISO DCR. In 6th international Conference on Language Resources and Evaluation (LREC 2008).

[19] Abinash Tripathy, Ankit Agrawal, and Santanu Kumar Rath. 2016. Classification of sentiment reviews using n-gram machine learning approach. Expert Systems with Applications 57 (2016), 117-126.

[20] Ingmar Visser and Maarten Speekenbrink. 2010. depmixS4: An R package for hidden Markov models. Fournal of Statistical Software 36, 7 (2010), 1-21. http: //www.jstatsoft.org/v36/i07/

[21] Margaret Wilson. 2002. Six views of embodied cognition. Psychonomic Bulletin \& Review 9, 4 (2002), 625-636. 